\section{Molecular diversity in a germplasm collection of avocado accessions from the tropical and subtropical regions of China}

\section{Yu Ge ${ }^{1,2}$, Fuchu Hu$u^{2}$, Lin $\operatorname{Tan}^{1}$, Bin $\mathrm{Wu}^{1}$, Tao Wang ${ }^{3}$, Teng Zhang ${ }^{4}$, Funing $\mathrm{Ma}^{1}$, Jianqiu $\mathrm{CaO}^{5}$, Zhining $\mathrm{Xu}^{1}$ and Rulin $\mathrm{Zhan}^{{ }^{*}}$}

\begin{abstract}
Avocado is an economically important crop and widely cultivated in the tropical and subtropical regions of China, whereas little is known about its genetic diversification. Twenty-six EST-SSR markers were developed based on avocado EST sequences. Molecular characterization was applied to assess the genetic relationships among different accessions. In total, nine gSSRs and nine EST-SSRs were selected to illuminate the genetic relationships within the 92 samples. Diversity parameters associated to the selected markers indicated the relatively low genetic diversity and a certain degree of inbreeding in the 92 avocado accessions. Clustering method and principal coordinate analysis clearly distinguished the accessions in accordance to the botanical races and geographic regions by molecular characterization. Moreover, the botanical race or hybrids of some unknown race accessions from different geographic origins could be determined based on known genotypes in the same cluster.
\end{abstract}

Keywords: Persea americana, botanical races, microsatellite markers, genetic diversity

\section{INTRODUCTION}

Avocado (Persea americana Mill.), belonging to the family Lauraceae of the order Laurales, is native to Mexico and Central and South America (Schaffer et al. 2012).Taxonomic treatments differ considerably in terms of the circumscription and defining of infraspecific avocado entities. Moreover, researchers have long considered that geographical isolation has likely resulted in the following three ecological races of avocado: Mexican ( $P$. americana var. drymifolia), Guatemalan (P. americana var. guatemalensis), and West Indian (P. americana var. americana) (Schaffer et al. 2012).Avocado is considered an significantly commercial crop in China, and is nowadays broadly grown and adequately utilized in some provinces such as Hainan, Guangdong, Guangxi, Yunnan, Guizhou, Fujian, and Taiwan in Southern China (Zhang et al. 2015, Ge et al. 2017a). The climate regions in these Chinese provinces comprise from subtropical to tropical, and conditions of the Chinese culture are well-suited for avocado cultivation (Zhang et al. 2015,Ge et al. 2017b).

The introduction and cultivation of avocado happen in 1918 in province of Taiwan, China, for the first time (Papademetriou 2000), followed by the clonally propagation of high quality cultivars and the development of avocados with premium botanical and horticultural traits for the processing industry.
Crop Breeding and Applied Biotechnology 19: 153-160, 2019 Brazilian Society of Plant Breeding. Printed in Brazil http://dx.doi.org/10.1590/198470332019v19n2a22

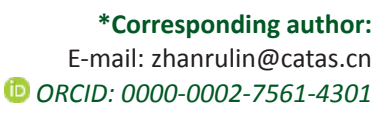

Received: 22 March 2017 Accepted: 15 January 2018

${ }^{1}$ Chinese Academy of Tropical Agricultural Sciences, Haikou Experimental Station, Hainan, People's Republic of China

${ }^{2}$ Hainan Academy of Agricultural Sciences, Key Laboratory of Tropical Fruit Tree Biology of Hainan Province, Hainan, People's Republic of China

${ }^{3}$ Liaoning Academy of Agricultural Sciences, Institute of Vegetable, Liaoning, People's Republic of China

${ }^{4}$ Tianjin derit seed industry Co. Ltd, Tianjing, People's Republic of China

${ }^{5}$ Hainan University, College of Horticulture and Landscape Architecture, Hainan, People's Republic of China 


\section{Y Ge et al.}

Since the late 1950s, abundant avocado cultivars are successively brought into China from the USA, Israel, Mexico, and other countries from Central America (Ge et al. 2017a). The selective breeding methods were initiated and continue up to now, principally maintained by some national scientific research bodies and other state and private farms, resulting in the breeding of more than ten superior avocado cultivars (Ge et al. 2017a). In addition, natural crossing among avocado cultivars usually happens, creating several new avocado hybrids on state and private farms. Some local varieties are also increasingly produced in somewhat remote area due to the distinct local geographical conditions. At present, several superior native Chinese avocado cultivars are broadly grown in the southern China, and the areas of avocado plantations have reached about three thousand hectares, with an average output of around $3 \mathrm{t} \mathrm{ha}{ }^{-1}$ (Zhang et al. 2015,Ge et al. 2017a).The avocado consumption in China has rapidly reached nearly $18^{\prime} 000 \mathrm{t}$ in 2016 ,compared to just over $30 \mathrm{t}$ in 2011(Ge et al. 2017a).

The avocado germplasm of these collections must be precisely identified to make it available for breeders all over the world (Schaffer et al. 2012). Molecular characterization is requisite for investigating the genetic relationship in avocado germplasm. Over the past two decades, some genetic relationship researches of avocado germplasm have been carried out with different types of molecular marker, containing RAPDs (random amplified polymorphic DNA) (Fiedler et al. 1998), VNTRs (variable number of tandem repeats) (Mhameed et al. 1997), RFLPs (restricted fragment length polymorphisms) (Furnier et al. 1990,Davis et al. 1998), SSRs (simple sequence repeats) (Ashworth and Clegg 2003, Schnell et al. 2003,Gross-German and Viruel 2013), and SNPs (single nucleotide polymorphism) (Chen et al. 2008, 2009).

Of the many DNA markers that have been developed, the SSRs, which are composed of repeated motifs between one and six nucleotide bases, are generally preferred by plant geneticist and breeder because they are broadly distribution in plant genomes, genetically multi-allelic, co-dominant, and well fitted for high-throughput genotyping (Ge et al. 2011). The gSSR (genomic simple sequence repeat) and EST-SSR (expressed sequence tag - simple sequence repeat) markers of avocado (Sharon et al. 1997,Ashworth and Clegg 2003,Borrone et al. 2007, Borrone et al. 2009) were developed and applied to investigate the genetic relationship in avocado accessions maintained in the USA (Ashworth and Clegg 2003, Schnell et al. 2003) and Spain (Alcaraz and Hormaza 2007, Gross-German and Viruel 2013), whereas, few researches have examined the genetic diversity in avocado germplasm from China.

This study investigated the different geographic origins of avocado germplasm from Southern China, and in a supplementary analysis, indicated a broadly and distinct visualization of genetic diversity and race identity in this avocado germplasm. This study is expected to inspire a marked improvement in avocado breeding.

\section{MATERIAL AND METHODS}

\section{Plant material}

Ninety-two avocado accessions used in this study, including commercial varieties and local Chinese selections, were selected as follows: 14 native avocado accessions from the Chinese Academy of Tropical Agricultural Sciences - CATAS, 20 from the Nanfeng State Farm - NFS, 7 from Xipei State Farm - XPS, 13 from Bayi State Farm - BYS, 6 from Xilian State Farm - XLS, 12 from Lanyang State Farm - LYS [Danzhou city (lat $19^{\circ} 31^{\prime} \mathrm{N}$, long $109^{\circ} 34^{\prime} \mathrm{E}$ and alt $20 \mathrm{~m}$ asl), Hainan Province, China]; 13 from the Daling State Farm - DLS [Baisha city (lat 19 $14^{\prime} \mathrm{N}$, long 109 $14^{\prime} \mathrm{E}$ and alt $60 \mathrm{~m}$ asl), Hainan Province, China]; 4 from the Mengmao State Farm - MMS [Ruili city (lat 24 $00^{\prime} \mathrm{N}$, long $97^{\circ} 50^{\prime} \mathrm{E}$ and alt $240 \mathrm{~m}$ asl), Yunnan Province, China]; and 3 from the Guangxi Vocational and Technical college - GVTC [Nanning city (lat $22^{\circ} 29^{\prime}$ $\mathrm{N}$, long $108^{\circ} 11 \mathrm{E}^{\prime}$ and alt $100 \mathrm{~m}$ asl), Guangxi Province, China]. The commercial sale of the commercial varieties has been authorized and permitted by the ministry of agriculture of China or departments of agriculture of the Chinese provinces, while the commercial sale of the local Chinese selections has not yet been authorized and permitted by the responsible government departments.

\section{Marker development and genotyping}

Avocado ESTs were acquired from the NCBI (http://www.ncbi.nIm.nih.gov/Entrez). Microsatellites within the expressed sequence tags were identified by the program SSRHunter (Li and Wang 2005). The repeat motifs were set as 1-6 bp, and the minimum number of repeats was $\geq 5$ for di-, tri-, tetra-, penta-, and hexa nucleotide. Primers were also designed through software Primer3 (http://primer3.ut.ee/). A total of 26 EST-SSR primer pairs were developed and 
designated as "CATASpa", to indicate Chinese Academy of Tropical Agricultural Sciences and Persea Americana (Tables 1 and 2). All primer pairs were produced by Genscript Biotech (Nanjing, China). Furthermore, 140 previously developed gSSR and EST-SSR primer pairs were utilized. Primer pairs with the prefix "SHRSPa" were developed by Borrone et al. 2009, "LMAV" by Gross-German and Viruel (2013), and "AV" by Sharon et al. (1997). Genomic DNA was extracted using the CTAB protocol as described by Ge et al. (2016), with minor modifications. Of the 92 accessions, five with higher phenotypic polymorphism were selected to filter out 166 developed gSSR and EST-SSR primer pairs. Polymerase chain reaction amplifications were conducted as described by Ge et al. (2011). The amplified fragments were separated on $6 \%$ denaturing polyacrylamide gels, which were stained as previously described (Sanguinetti et al. 1994).

\section{Data analysis}

For the gSSR and EST-SSR molecular analyses, only strong, reproducible and clearly distinguishable bands were used and putative alleles were scored on a 1-7 scale, according to the band sizes for each primer pair. The genetic information was investigated for nine gSSRs and nine EST-SSRs, based on the following parameters: number of alleles per locus $(A)$; observed heterozygosity (Ho); expected heterozygosity $\left(H_{e}=1-\Sigma \mathrm{p}_{\mathrm{i}}^{2}\right)$, where $p_{\mathrm{i}}$ is the frequency of the $i^{\text {th }}$ allele (Nei 1987); effective number of alleles $\left(N_{e}=1 /\left(1-N_{e}\right)\right.$; Wright's fixation index (F); the polymorphic information content (PIC) calculated for each marker as $P I C_{i}=1-\sum p_{i j}^{2}$, where $p_{i j}$ is the frequency of the $j^{\text {th }}$ allele in clone $i$. Software GenAlEx v6.5 (Peakall and Smouse 2012) was used to calculate A, Ho, He, Ne, PIC, and principal coordinate analysis (PCoA). Cluster analysis was performed with PowerMarker v3.25 (Liu and Muse 2005), a neighbor-joining tree was constructed based on the shared alleles, and software MEGA6.0 was used to visualize the phylogenetic tree (Tamura et al. 2013).

\section{RESULTS AND DISCUSSION}

The 92 avocado accessions comprised 17 commercial varieties and 75 local Chinese selections collected on two agricultural science research units and seven state farms. Fourteen of the 17 commercial varieties came from CATAS, while only 3 commercial varieties were from the GVTC. Seventy-one of the 75 local Chinese selections were artificially selected on six state farms in Hainan Province, whereas the other four local Chinese selections (RLW-1, RLW-2, RLW-3, and RLW-4) from the MMS in Yunnan Province were the result of natural unmonitored and unmanipulated selection. Moreover, 18 Mexican accessions, 28 Guatemalan accessions, 10 interracial hybrids, and 36 unknown race accessions were represented in the total of 92 avocado accessions. The Mexican accessions were mainly collected from NFS and XPS. Most of the Guatemalan accessions were from CATAS, BYS, DLS, and GVTC. The interracial hybrids came from CATAS and LYS, and all unknown race accessions from all seven state farms.

From a total of 3089 avocado EST sequences, 278 (9\%) contained the SSR motif. A total of 26 EST-SSR markers were ultimately developed. Thirteen EST-SSRs contained the AG/ TC motif, six the AT/TA motif and seven other motifs. The number of repeats varied between 5 and 12 (Table 1). The proportion of avocado EST sequences containing the SSR motif at present was higher (9\%) than that reported by Gross-German and Viruel (2013) (4\%).

A set of two Guatemalan accessions (RN-5 and RN-6), two interracial (Guatemalan $\times$ Mexican) hybrids (RN-1-GM

Table 1. Description of the 26 EST-SSRs

\begin{tabular}{|c|c|c|c|}
\hline Primer name & Repeat motif & $T_{a}\left({ }^{\circ} \mathrm{C}\right)$ & Expected length (bp) ${ }^{a}$ \\
\hline CATASpa-01 & $(A G) 6$ & 60 & 150 \\
\hline CATASpa-02 & $(G A) 5$ & 59 & 237 \\
\hline CATASpa-03 & (TC)5(GGA)7 & 59 & 218 \\
\hline CATASpa-04 & $(A G) 6$ & 59 & 169 \\
\hline CATASpa-05 & $(\mathrm{CT}) 5$ & 57 & 227 \\
\hline CATASpa-6 & (AT)5 & 54 & 224 \\
\hline CATASpa-07 & (AT)5 & 54 & 231 \\
\hline CATASpa-08 & (ATC)6 & 57 & 221 \\
\hline CATASpa-09 & (AT)5 & 60 & 272 \\
\hline CATASpa-10 & $(\mathrm{TC}) 6$ & 57 & 209 \\
\hline CATASpa-11 & (AG)5 & 56 & 202 \\
\hline CATASpa-12 & $(\mathrm{CT}) 7$ & 53 & 165 \\
\hline CATASpa-13 & $(\mathrm{CT}) 5$ & 59 & 223 \\
\hline CATASpa-14 & (TA)5 & 57 & 223 \\
\hline CATASpa-15 & $(\mathrm{GA}) 11$ & 57 & 243 \\
\hline CATASpa-16 & (CA)6 & 58 & 168 \\
\hline CATASpa-17 & (TC)5 & 56 & 173 \\
\hline CATASpa-18 & $(G A) 5$ & 57 & 202 \\
\hline CATASpa-19 & $(\mathrm{TC}) 6$ & 57 & 188 \\
\hline CATASpa-20 & (AT)5 & 59 & 227 \\
\hline CATASpa-21 & (AT)5 & 57 & 243 \\
\hline CATASpa-22 & (CA)5 & 57 & 220 \\
\hline CATASpa-23 & $(\mathrm{CCA}) 7$ & 57 & 247 \\
\hline CATASpa-24 & $(\mathrm{GA}) 7$ & 58 & 238 \\
\hline CATASpa-25 & (TAT) 5 & 58 & 223 \\
\hline CATASpa-26 & (ATAA) 5 & 59 & 176 \\
\hline
\end{tabular}

$T_{a}$ : optimum annealing temperature; ${ }^{\text {a }}$ Length determined on the basis of the expressed sequence 
and RN-2-GM), and one Mexican accession (RN-16), presenting the different ecotypes, were applied to screen $166 \mathrm{gSSRs}$ and EST-SSRs. Of these, nine gSSRs and nine EST-SSRs were selected to assess the genetic relationship within the 92 accessions on account of testing standard. Diversity parameters associated to the said nine gSSRs and nine EST-SSRs were listed in Table 2 and Table 3. A total of 38 and 30 alleles were revealed by nine gSSRs and nine EST-SSRs, respectively, with an average of 4.22 alleles per gSSR and 3.33 alleles per EST-SSR. The PIC values, representing allele frequency and diversity between these accessions, were on average 0.50 per gSSRs and 0.47 per EST-SSRs, respectively. The effective number of alleles of the gSSRs varied from a low value of 1.48 for AVD003II to a high value of 4.08 for AVO102, with an average value of 2.52. The EST-SSRs ranged from a low of 1.64 for CATASpa-24 to a high of 3.16 for SHRSPa197, with an average value of 2.29. The SSR markers were applied previously to ascertain genetic diversity among the Mexican, Guatemalan, and West Indian ecotypes and interracial hybrids (Ashworth and Clegg 2003, Schnell et al. 2003,Alcaraz and Hormaza 2007, Gross-German and Viruel 2013). These studies reported that the average number of alleles per SSRs varied from 9.75 to 18.8 , which was higher than found in this study. This provided evidence that the genetic diversity of the avocado accessions in this study was relatively low. This could be due to the fact that 75 out of 92 avocado accessions in this study belong to local Chinese avocado selections and wild varieties, which have gradually developed in relatively isolated production zones without seed exchange among the local unique geographical environments of the state farms of China, forming groups with similar genetic background. The relatively low genetic diversity of the avocado accessions in this study was also indirectly confirmed by the observed and expected heterozygosity.

The $\mathrm{Ho}$ of the gSSRs varied from 0.00 to 0.32 (mean of 0.08 ) and the EST-SSRs ranged from 0.00 to 0.14 (mean of 0.04). The He of the gSSRs ranged from 0.32 to 0.76 (mean of 0.57 ) and the EST-SSRs from 0.39 to 0.68 (mean of 0.54 ). The two parameters were compared based on the $\mathrm{F}$ value. For 18 markers, this parameter presented positively with an overall mean of all gSSRs and EST-SSRs of 0.88 , displaying an overall heterozygote deficiency. These results indicated inbreeding to a certain degree in the avocado accessions in this study. This finding was in line with observations described

Table 2. Primer sequences of the 26 EST-SSRs

\begin{tabular}{|c|c|c|}
\hline Primer name & Forward primer $\left(5^{\prime} \rightarrow 3^{\prime}\right)$ & Reverse primer $\left(5^{\prime} \rightarrow 3^{\prime}\right)$ \\
\hline CATASpa-01 & ATTGGAAGGCGGATAGGAGG & GCCCCACАСТTCTTCTCTCT \\
\hline CATASpa-02 & GCCTGATTCTGATGCAAGGG & TACAAACAATGCGTGAGGGC \\
\hline CATASpa-03 & ATCTGGTGGGGAACTTGGAG & СССТСCTCATCAAGTCCTCC \\
\hline CATASpa-04 & СТTССТСТGСААТСТССССА & TGCAGACAAAGGAGCCATTG \\
\hline CATASpa-05 & СССТСТСТСТСАСААААСGC & GAGAGGCCGATGTAGAGCTT \\
\hline CATASpa-6 & GGAAGCCACCACCGAAATAC & AACTGCCGTAACAAAGCGTG \\
\hline CATASpa-09 & TCAATGTAAAGACTGGGGAGACT & CTTGAGGCTTGGAGTTCACA \\
\hline CATASpa-10 & AGGAACGGATGGATCGGATC & TCCCCAATATCTTGTGCATTCG \\
\hline CATASpa-11 & AGGGAAACGGTATGCAAGGA & TGAATGGGCTGGGTATCTCC \\
\hline CATASpa-12 & AGGGAAACTGTGCTCAAGGT & TGACCCACCACAATCTTCCA \\
\hline CATASpa-13 & GCCCACСTCTTTCTTCCTCT & TTATCGAGGGCAGCATGGAA \\
\hline CATASpa-17 & AAAGCAACCGAAACGATCCC & AATGCTAACTGAAGAACCCTCT \\
\hline CATASpa-18 & ATGCCAAAAGGCCAACAGAG & CTAAAGTTTCCTGGCCCAGC \\
\hline CATASpa-19 & ATTGACGCCATATTCTCCGC & TCGAGGCGACAGATGAATGA \\
\hline CATASpa-20 & ACCAATCTCTGTGCCATCCA & AGAGAAAATCACATGTCCCCAT \\
\hline CATASpa-21 & ATATGGGAAGCCACCACCAA & ACATAAAAGCTGCTCCACGT \\
\hline CATASpa-22 & ACAGATCTTATCGCCTGAGC & GGTTCTTCTGACCGTTTCTCA \\
\hline CATASpa-23 & ATCGAACCCCAATCACTCGT & GCTGGGTCTCGTAGGTCTTT \\
\hline CATASpa-24 & AAAACCCAGGGCTAGAGGAC & CGATGACTTTGGTTTTCGCC \\
\hline CATASpa-25 & GAGTGCAAACAAACAATCCCT & GCCAGTCTGATTTGCCCTTC \\
\hline CATASpa-26 & GGCCATTTGATTCCCTCTTCC & GGTCACCCAGGGATCATCTC \\
\hline
\end{tabular}


in other studies on avocado genotypes using SSRs and ESTSSRs (Schnell et al. 2003,Alcaraz and Hormaza 2007, Borrone et al. 2007, Gross-German and Viruel 2013).

In addition, the newly developed EST-SSRs in this study had a lower level of polymorphism than the gSSRs. This finding is outstanding since the EST-SSRs lied in the coding regions experience strong selective pressure and hence often produce few variations. A lower polymorphic value of EST-SSRs than of gSSRs was also reported for Brassica rapa (Ge et al. 2011).

For the SSR analysis, genetic distances among all 92 avocado accessions ranged from 0.00 to 0.30 . Cluster analysis clustered the 92 accessions into two major groups, with a genetic distance of 0.30 (Figure 1). The dendrogram demonstrated a clear separation between Guatemalan accessions and Guatemalan $\times$ Mexican hybrids from the Mexican accessions. In cluster I, the 62 accessions were clustered into two sub-clusters (genetic distance = 0.295); sub-cluster I-I was composed of 44 Guatemalan accessions, and divided into four groups consistent with their geographical origins. Group I-I-I contained three Guatemalan varieties from GVTC, and group I-I-II two Guatemalan varieties from CATAS and 13 Guatemalan/ local Chinese selections and unknown race accessions from DFS. Two Guatemalan varieties from CATAS and 13 Guatemalan /local Chinese selections and unknown race accessions from BYS formed group I-I-III, while group I-IIV included five Guatemalan varieties from CATAS and six Guatemalan /local Chinese selections and unknown race accessions from XLS. Sub-cluster I-II included 18 Guatemalan $\times$ Mexican hybrids and unknown race accessions, and was divided into two groups consistent with their geographical origins. Group I-II-I comprised two Guatemalan × Mexican hybrids from CATAS and 12 Guatemalan $\times$ Mexican hybrids and unknown race accessions from LYS, and group I-II-II was composed of four unknown wild races from MSF. In cluster II, the 30 accessions were grouped independently into two sub-clusters (genetic distance $=0.28$ ); sub-cluster II-I was clearly separated into two groups that reflected geographical origins. Group II-I-I contained 20 Mexican/ local Chinese selections and unknown race accessions from NFS, while group II-I-II consisted of seven Mexican/local Chinese selections and unknown race accessions from XPS. Sub-cluster II-II comprised only three Mexican varieties from CATAS. In addition, cluster analysis demonstrated genetic distances of 0.00 between some avocado accessions, e.g., between the accession NFS-11, NFS-12, NFS-13, and NFS-

14 and NFS. Molecular characterization may show that these four accessions are identical. Therefore, morphological characterization to analyze the genetic distance combined with molecular characterization is required.

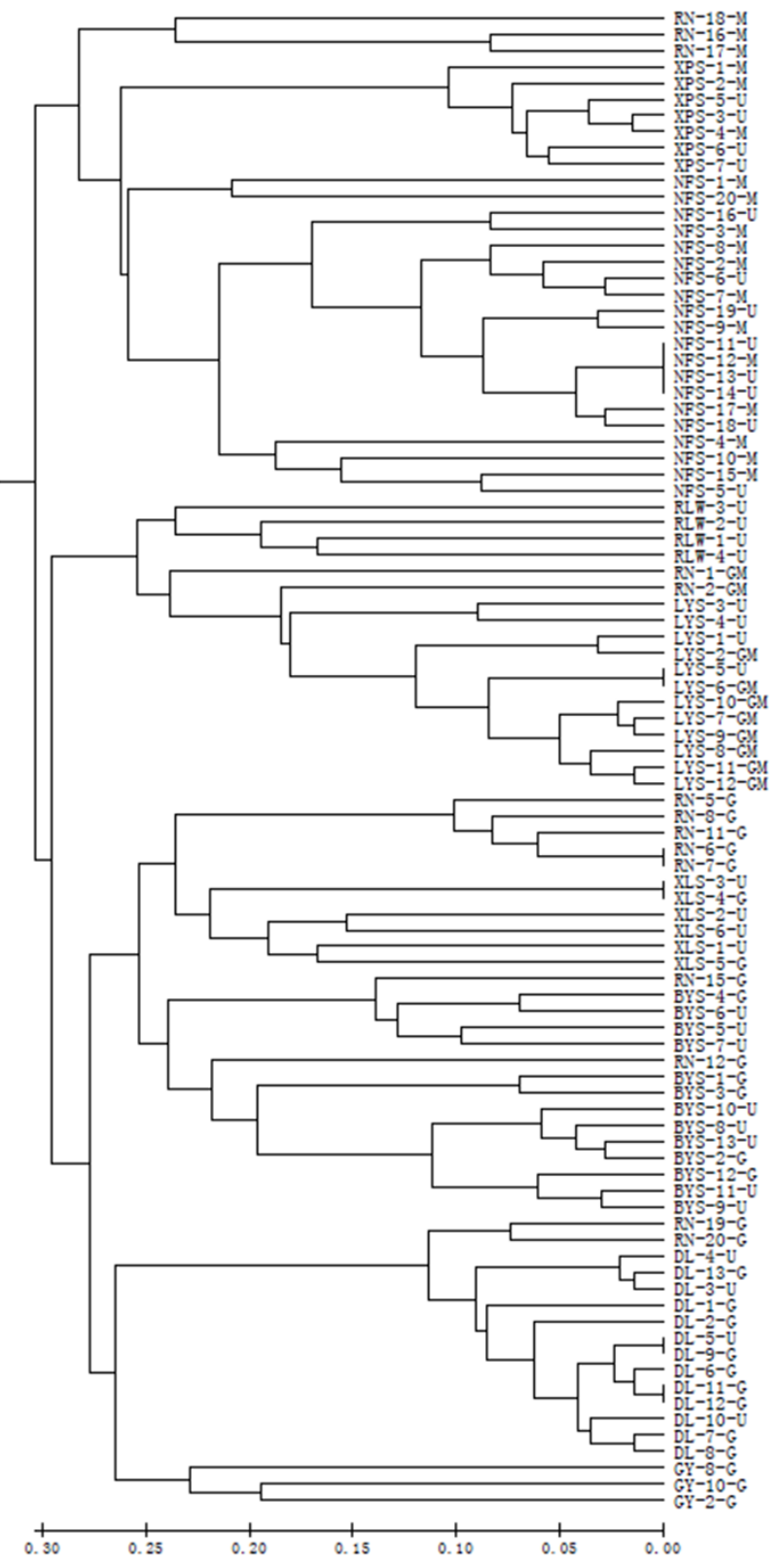

Figure 1. Neighbor-joining tree calculated for the 92 avocado accessions studied based on shared alleles for the 18 SSR and EST-SSR markers. The avocado accessions were labelled by the code + race (abbreviation). RN: Renong; XPS: Xipei State Farm; NFS: Nanfeng State Farm; RLW: Ruili wild; LYS: Lanyang State Farm; XLS: Xilian State Farm; BYS: Bayi State Farm; DL: Daling; GY: Guangxi; G: Guatemalan; M: Mexican; GM: Interracial hybrids between Guatemalan and Mexican; U: Unknown. 
Figure 2 demonstrated the clustering situation of the 92 accessions on the first two principal coordinate values through PCoA. On the basis of the first coordinate, which possessed $18.29 \%$ of the total variation, the accessions were distinctly clustered into two sub-clusters. The Guatemalan accessions were distinguished from Mexican accessions and Guatemalan $\times$ Mexican hybrids. On the basis of the second coordinate, which occupying $13.00 \%$ of the total variation, we observed that the Mexican accessions were distinguished from Guatemalan $\times$ Mexican hybrids.

The greater genetic relationship between Guatemalan and Mexican accessions at the interspecies than intraspecies level on account of SSR marker features in this study was in accordance with previous studies (Ashworth and Clegg 2003,

Table 3. Diversity parameters associated to the nine SSRs and nine EST-SSRs analyzed in the 92 avocado accessions

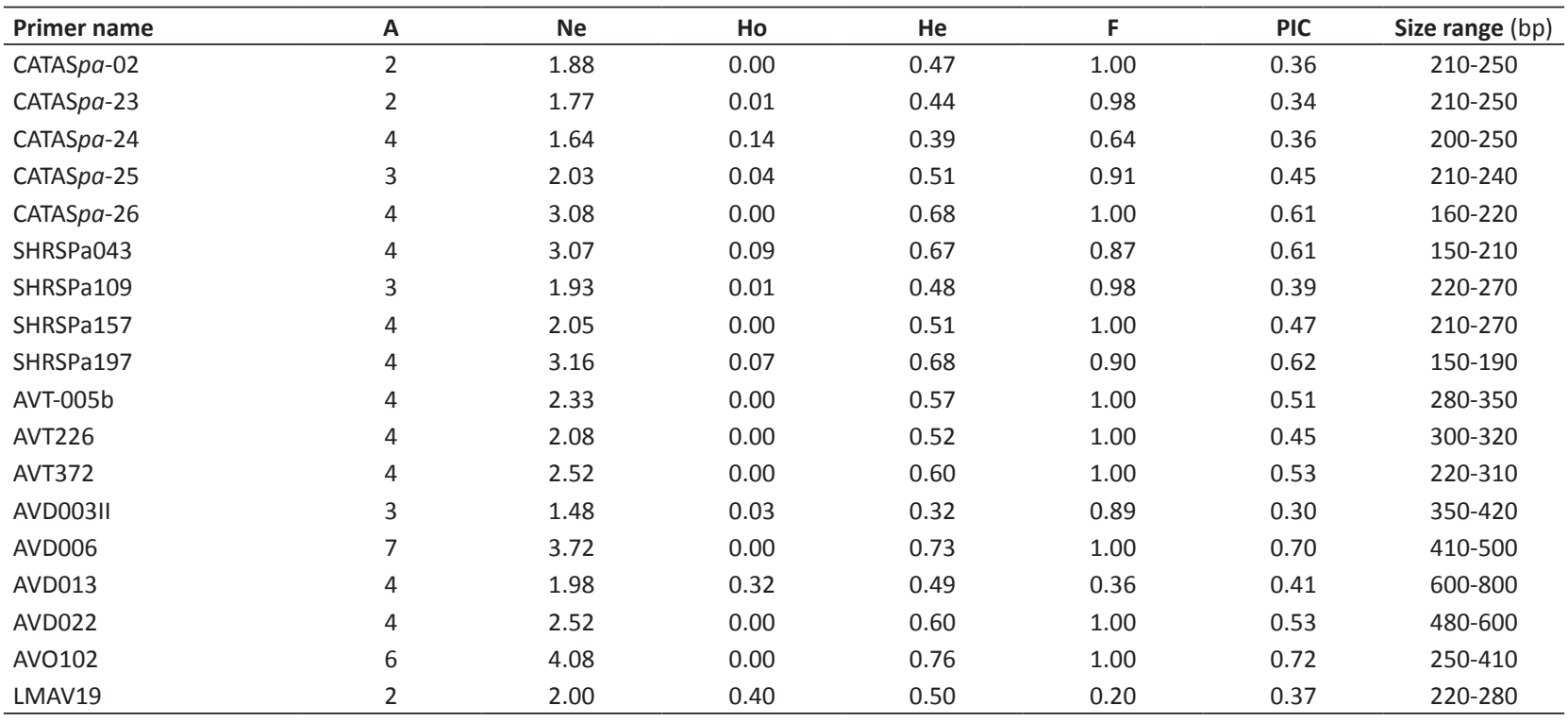

A: total number of alleles; Ne: effective number of alleles; Ho: observed heterozygosity; He: expected heterozygosity; F: Wright's fixation index; PIC: polymorphic information content.

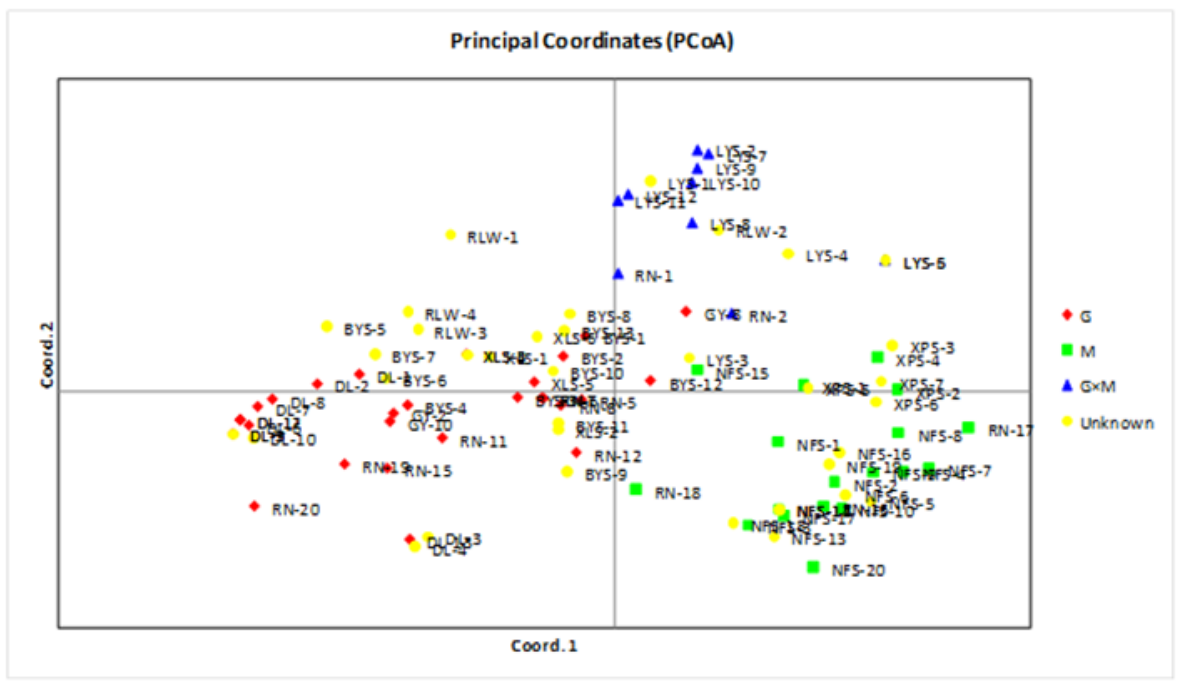

Figure 2. Principal coordinate analysis of the 92 avocado accessions based on genetic distances calculated from gSSR and EST-SSR allelic data. RN: Renong; XPS: Xipei State Farm; NFS: Nanfeng State Farm; RLW: Ruili wild; LYS: Lanyang State Farm; XLS: Xilian State Farm; BYS: Bayi State Farm; DL: Daling; GY: Guangxi; Red rhombus: Guatemalan; Green square: Mexican; Purple triangle: Interracial hybrids between Guatemalan and Mexican; Yellow roundness: Unknown. 
Schnell et al. 2003, Alcaraz and Hormaza 2007, Gross-German and Viruel 2013). Besides, discrimination in this study normally clustered the different accessions according to the geographic origin, while the finding that some accessions derived from different geographic origins joined into the same sub-cluster was also reported. For example, several Guatemalan, Guatemalan $\times$ Mexican hybrids, and Mexican varieties from CATAS were clustered with some local Chinese selections and unknown race accessions from different state farms in the municipality of Danzhou, Hainan province (Figure 2). One factor leading to this promiscuous clustering event was the occurrence of cultivar exchange among researchers and breeders since the late 1980s. The CATAS, as a national scientific research unit, was commissioned to popularize superior avocado varieties to breeders on adjacent state farms. Some commercial cultivars from CATAS may be the male or female parent of some local Chinese selections and unknown race accessions from different state farms in Danzhou, Hainan, which could be inferred from the results of our study (Figure 2). In addition, the botanical races or hybrids of some unknown race accessions from different geographic origins could be determined based on known genotypes in the same group. For example, DL-3, DL-4, DL-5, and DL-10 could belong to Guatemalan /local Chinese selections based on other known Guatemalan/local Chinese selections in the same group.

\section{CONCLUSIONS}

A total of 26 EST-SSR markers were produced according to EST sequences of avocado. The 92 avocado accessions (18 Mexican and 28 Guatemalan accessions, 10 interracial hybrids, and 36 unknown race accessions) were applied to assess the genetic relationships based on nine gSSRs and nine EST-SSRs. The diversity parameters of these suggested that the genetic diversity of 92 avocado accessions was relatively low and indicated a certain degree of inbreeding. The 92 accessions of different botanical races and from different geographic regions were distinctly differentiated by cluster analysis and principal coordinates analysis. Moreover, the botanical race or hybrids of some unknown race accessions from different geographic origins could be determined based on known genotypes in the same cluster.

\section{ACKNOWLEDGMENTS}

This study was supported by the Open Foundation of Key Laboratory of Tropical Fruit Tree Biology of Hainan Province (Grant No. KFZX2017001) and the National Natural Science Foundation of China (Grant No. 31701883).

\section{REFERENCES}

Alcaraz ML and Hormaza JI (2007) Molecular characterization and genetic diversity in an avocado collection of cultivars and local Spanish genotypes using SSRs. Hereditas 144: 244-253.

Ashworth VETM and Clegg MT (2003) Microsatellite markers in avocado (Persea americana Mill.) genealogical relationships among cultivated avocado genotypes. Journal of Heredity 94: 407-415.

Azevedo AON, Azevedo CDO, Santos PHAD, Ramos HCC, Boechat MSB, Arêdes FAS, Ramos SRR, Mirizola LA, Perera L, Aragão WM and Pereira MG (2018) Selection of legitimate dwarf coconut hybrid seedlings using DNA fingerprinting. Crop Breeding and Applied Biotechnology 18: $409-416$.

Borrone JW, Brown JS, Tondo CL, Mauro-Herrera M, Kuhn DN, Violi HA, Sautter RT and Schnell RJ (2009) An EST-SSR-based linkage map for Persea americana Mill. (avocado). Tree Genetics and Genomes 5: 553-560.

Borrone JW, Schnell RJ, Violi HA and Ploetz RC (2007) Seventy microsatellite markers from Persea americana Miller (avocado) expressed sequence tags. Molecular Ecology Notes 7: 439-444.

Chen H, Morrel PL, Ashwoth VETM, De la Cruz M and Clegg MT (2008) Nucleotide diversity and linkage disequilibrium in wild avocado
(Persea americana Mill.). Journal of Heredity 99: 382-389.

Chen H, Morrel PL, Ashwoth VETM, De la Cruz M and Clegg MT (2009) Tracing the geographic origins of mayor avocado cultivars. Journal of Heredity 100: 56-65.

Davis J, Henderson D and Kobayashi M (1998) Genealogical relationships among cultivated avocado as revealed through RFLP analysis. Journal of Heredity 89: 319-323.

Ferreira F, Scapim CA, Maldonado C and Mora F (2018?]SSR-based genetic analysis of sweet corn inbred lines using artificial neural networks. Crop Breeding and Applied Biotechnology 18: 309-313.

Fiedler J, Bufler G and Bangerth F (1998) Genetic relationships of avocado (Persea americana Mill.) using RAPD markers. Euphytica 101: 249-255.

Furnier GR, Cummings MP and Clegg MT (1990) Evolution of the avocados as revealed by DNA restriction site variation. Journal of Heredity 81: 183-188.

Ge Y, Lin XE, Wang JS, Zang XP and Ma WH (2016) Comparative study of DNA extraction methods for the pulp of avocado (Persea americana Mill.). Chinese Journal of Tropical Agriculture 36: 33-36.

Ge Y, Ramchiary N, Wang T, Liang C, Wang N, Wang Z, Choi SR, Lim YP and Piao ZY (2011) Development and linkage mapping of unigene- 


\section{Y Ge et al.}

derived microsatellite markers in Brassica rapa L. Breeding Science 61: 160-167.

Ge Y, Si XY, Cao JQ, Zhou ZX, Wang WL and Ma WH (2017b) Morphological characteristics, nutritional quality, and bioactive constituents in fruits of two avocado (Persea americana) varieties from Hainan province, China. Journal of Agricultural Science 9: 8-17.

Ge Y, Si XY, Lin XE, Wang JS, Zang XP and Ma WH (2017a) Advances in avocado (Persea americana Mill.). South China Fruit 46: 63-70.

Gross-German E and Viruel MA (2013) Molecular characterization of avocado germplasm with a new set of SSR and EST-SSR markers: genetic diversity, population structure, and identification of racespecific markers in a group of cultivated genotypes. Tree Genetics and Genomes 9: 539-555.

Hou MY, Mu GJ, Zhang YJ, Cui SL, Yang XL and Liu LF (2017) Evaluation of total flavonoid content and analysis of related EST-SSR in Chinese peanut germplasm. Crop Breeding and Applied Biotechnology 17: 221-227.

Li Q and Wang JM (2005) SSRHunter: development of a local searching software for SSR sites. Hereditas 27: 808-810.

Liu K and Muse SV (2005) PowerMarker: an integrated analysis environment for genetic marker analysis. Bioinformatics 21: 21282129.

Mhameed S, Sharon D, Kaufman D, Lahav E, Hillel J, Degani C and Lavi U (1997) Genetic relationships within avocado (Persea americana Mill.) cultivars and between Persea species. Theoretical and Applied Genetics 94: 279-286.
Nei M (1987) Molecular evolutionary genetics. Columbia University Press, New York, p. 22-25.

Papademetriou MK (2000) Avocado Production in Asia and the Pacific. FAO Publisher, Bangkok, 35p.

Peakall R and Smouse PE (2012) GenAIEx 6.5: genetic analysis in Excel. Population genetic software for teaching and research - an update. Bioinformatics 28: 2537-2539.

Sanguinetti CJ, Dias Neto E and Simpson AJ (1994) Rapid silver staining and recovery of PCR products separated on polyacrylamide gels. Biotechniques 17: 914-921.

Schaffer B, Wolstenholme BN and Whiley AW (2012) The avocado: botany, production and uses. $2^{\text {nd }}$ edn, CPI Group, Croydon, p. 31-32.

Schnell RJ, Brown JS, Olano CT, Power EJ, Krol CA, Kuhn DN and Motamayor JC (2003) Evaluation of avocado germplasm using microsatellite markers. Journal of the American Society for Horticultural Science 128: 881-889.

Sharon D, Cregan PB, Mhameed S, Kusharska K, Hillel J, Lahav E and Lavi $U$ (1997) An integrated genetic linkage map of avocado. Theoretical and Applied Genetics 95: 911-921.

Tamura K, Stecher G, Peterson D, Fillipski A and Kumar S (2013) MEGA6: molecular evolutionary genetics analysis version 6.0. Molecular Biology and Evolution 30: 2725-2729.

Zhang L, Zhang DS and Liu KD (2015) Environmental analysis and countermeasures for industrial development of Hainan avocado. Chinese Journal of Agricultural Resources and Region Planning 36: 78-84. 